Pernet, C. J. (1957). J. gen. Microbiol. 16, 250-264,

\title{
An Apparatus for the Continuous Culture of Bacteria at Constant Population Density
}

\author{
By C. J. PERRET \\ National Institute for Medical Research, Mill Hill, London, N.W. 7, and now \\ Lister Institute of Preventive Medicine, Chelsea Bridge Road, London, S.W. 1
}

SUMMARY: A self-regulating continuous culture apparatus is described, designed for studying growth and enzyme production in bacteria which require complex media; it might also be suitable for the cultivation of suspended tissue cells. The growth vessel is a tilted cardioid-shaped flask spinning about its long axis. Aeration and mixing are achieved in such a manner that no frothing occurs and no 'anti-foam' agents are required. Adherent bacterial colonies do not form on the walls of the growth vessel. Samples are removed rapidly under aerobic conditions. The basal medium can be automatically augmented with special metabolites at constant concentration without contaminating the main medium reservoir. The apparatus is autoclaved in one unit after assembly. Factors governing the design of similar small-scale apparatus are discussed.

The fundamental theory of homocontinuous or capacity flow culture has already been established by numerous authors (Monod, 1950; Novick \& Szilard, 1950 $a, b$; Spicer, 1955; Herbert, Elsworth \& Telling, 1956), and the principles governing the construction of a self-regulating apparatus for maintaining growing bacterial cultures at constant population density are generally agreed. The three basic requirements for aerobic organisms are: a growth vessel in which the culture is thoroughly mixed and aerated; a reservoir to supply fresh medium to the growth vessel at constant rate; a device to keep the volume of culture in the growth vessel constant, by removing culture at the same rate as the addition of fresh medium. However, since various ancillary devices are necessary if the apparatus is to operate accurately without too much attention, and since most operations must be performed at constant temperature under aseptic conditions, the final assembly is rather more complicated than its simple formulation suggests.

Each of the practical difficulties which arise can be resolved in various ways, and numerous assemblies for continuous culture have already been described (see the review by Novick, 1955; also De Haan \& Winkler, 1955; Herbert et al. 1956), but none of them is the perfect all-purpose equipment. When designing a continuous culture method it is therefore necessary to take into account the particular purpose for which it is to be used. An excellent apparatus for mass-production of bacteria may be quite unsuitable for studies of mutation rates or cell metabolism. The pathogenicity of the organism, its nutritional requirements and growth rate also affect the design of the apparatus.

The apparatus described here was designed primarily for studying the induced formation of penicillinase in broth cultures of Bacillus cereus at growth 
densities equivalent to 1-2 mg. dry wt. organisms $/ \mathrm{ml}$. culture. The proposed experiments demanded the following conditions: (1) easy sterilization and the ability to operate with little chance of contamination; (2) low medium consumption; (3) aeration without foaming; (4) absence of adherent bacterial colonies from the walls of the growth vessel; (5) contact of medium and culture only with chemically inert materials, such as glass and silicone; (6) rapid transfer of culture from growth vessel to sampling tube under aerobic conditions; (7) augmentation of the basal medium with special metabolites during operation, without contamination of the main medium reservoir.

A prototype apparatus was completed to these specifications in 1951 and has since worked satisfactorily. Details of its performance and the experimental results will be given in another paper. However, experience suggested improvements which have been incorporated in later models, and which are mentioned here after the description of the prototype. Finally, some of the factors governing the design of the components are discussed for the guidance of workers who may wish to adapt the equipment for their own purpose. It appears that similar apparatus might be particularly suitable for the continuous culture of suspended tissue cells.

\section{GENERAL DESCRIPTION}

The prototype apparatus is intended for operation in a hot-room, and is mounted on a non-rusting metal framework in such a manner that all parts requiring sterilization can be autoclaved in one unit after assembly. All glass components are made of 'Pyrex' glass with standard joints lubricated with silicone grease.

Fig. 1 shows in schematic form the functions of the various components and their interconnexions; Fig. 2 is a conventionalized diagram of the apparatus lettered to correspond with the detailed description given below; Fig. 3 shows details of the growth vessel; and Fig. 4 the associated electrical circuits.

\section{Air supply}

Medium enters the growth vessel under gravity and is removed by suction; since the growth vessel has a liquid seal, the air inside it must be at nearly atmospheric pressure. The apparatus therefore has two air-circuits; one operates above atmospheric pressure and supplies sterile humidified air, and the other operates below atmospheric pressure and withdraws air. These are referred to respectively as the positive and negative air lines: they are constructed of wide-bore tubing to eliminate flow pressure gradients, except at points where a constriction is inserted for some specific purpose.

$P$ ositive air line. Compressed air passes through a reservoir $Q$ to the main filter $\boldsymbol{P}$ which is a $5 \mathrm{~cm}$. diam. tube packed firmly with non-absorbent cotton wool. From $P$ the air by-passes a combined blow-off valve and water manometer $O$, consisting of a wash-bottle with the inlet tube dipping $20 \mathrm{~cm}$. into 
water, and flows to the saturator $\boldsymbol{M}$. The saturator is in effect a wash-bottle, with a $60 \mathrm{~cm}$. neck serving as a reflux condenser. The incoming air is bubbled through water in a flask maintained at about $70^{\circ}$ by a thermostatically-

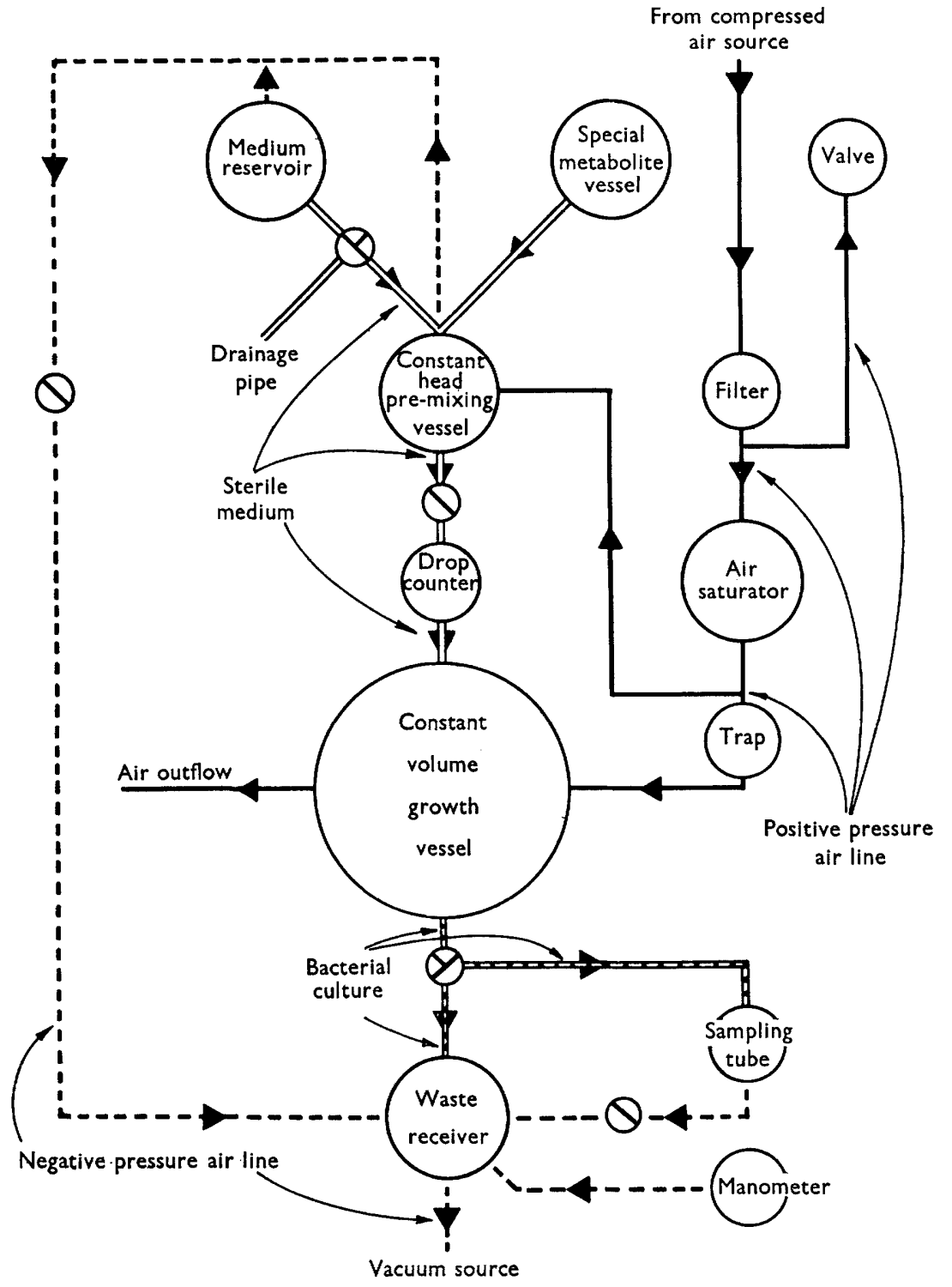

Fig. 1. Schematic diagram of the apparatus, showing the functions of the components and their interconnexions.

controlled heating mantle, and then cooled to ambient temperature as it rises up the neck. When necessary the flask can be topped-up with sterile water through a side-arm $N$. 
A small proportion of the humidified sterile air emerging from $M$ is diverted to operate the constant-head device in $B$; the majority passes to the $\operatorname{trap} L$ which is again a wash-bottle with the inlet tube immersed in water to a depth

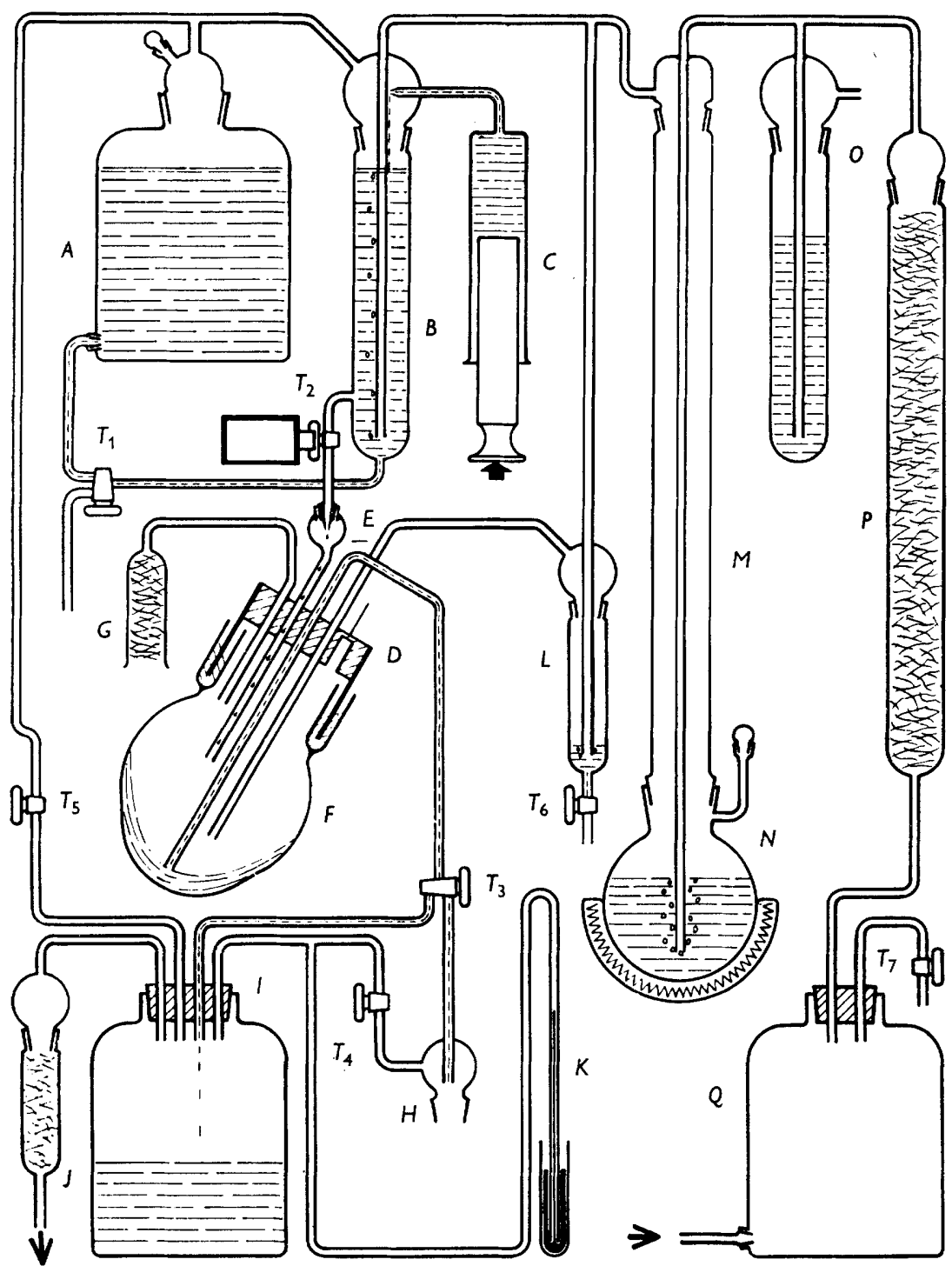

Fig. 2. Conventionalized diagram of the apparatus lettered to correspond with the description in the text. The components are not drawn to scale.

of $1 \mathrm{~cm}$. only. If extra water collects in the trap it can be run off through $\operatorname{tap} T_{6}$.

From the trap, air passes through the fixed sealing-head $D$ to the growth vessel $F$, where it is blown over the surface of the culture. Most of the air is 
then sucked out with excess culture through the constant-volume control tube, but some passes directly into the atmosphere through a large area cotton-wool, filter $G$.

Negative air line. A slight suction is maintained through a cotton-wool filter $J$, which removes aerosols, to a $10 \mathrm{l}$. aspirator $I$ serving both as a lowpressure reservoir and as a receptacle for unwanted effluent culture from the growth vessel. Lysol is placed in the bottom of $I$ to prevent further bacterial growth.

From $I$ negative air lines run directly to a mercury manometer $K$, through a tap $T_{4}$ to the sampling head $H$ and through a three-way $\operatorname{tap} T_{3}$ to the constantvolume control tube in the growth vessel. A fourth connexion is made through a variable leak $T_{5}$, which is a grooved tap of the type shown in Fig. 5, to the medium reservoir $A$ and constant-head vessel $B$.

\section{Medium supply}

The medium reservoir $A$ is a 5 l. graduated aspirator, closed at the top by a head connected to the negative air line and carrying a stoppered refilling port through which the reservoir may be replenished while the apparatus is working. Medium flows from $\boldsymbol{A}$ under gravity through a tube fitted with a three-way drainage tap $T_{1}$ at its lowest point, entering at the bottom of the constant-head vessel $B$ in which it rises to the same level as the liquid in $A$.

The constant-head vessel is a graduated tube of about $300 \mathrm{ml}$. capacity, slightly longer than the height of $\boldsymbol{A}$. It is fitted with a wash-bottle head which is so connected to the positive and negative air lines that a slow stream of sterile air bubbles is drawn through the medium. The pressure at the tip of the bubbling tube is fixed above that in the growth vessel by the depth of water in $\operatorname{trap} L$; i.e. about $+1 \mathrm{~cm}$. of water. The assembly operates as a modified Mariotte bottle supplying medium at a constant head to $T_{2}$ and is able to compensate rapidly for ambient temperature or pressure changes. In addition, the bubbles stir the contents of the vessel and mix any special metabolites being injected from $C$.

The special metabolite vessel $C$ is a $100 \mathrm{ml}$. 'Pyrex' glass syringe driven by a pulsed stepwise motor through a screw thread. One pulse to the motor turns the screw through $\frac{1}{120}$ revolution and injects $0.003 \mathrm{ml}$. of metabolite solution through a fine capillary into $B$. Pulses are generated electrically at intervals continuously variable from $\mathbf{0 . 5}$ to 50 sec., and also at a fixed short interval of $0.05 \mathrm{sec}$.

Medium leaves $B$ through a side-arm, in order to avoid any precipitate which may have collected at the bottom of the vessel, and flows under gravity to the adjustable flow resistance $T_{2} . T_{2}$ is a glass tap made leaky by cutting a pair of shallow circumferential grooves in its surface with a sharp triangular needle file (Fig. 5). From $T_{\mathbf{2}}$ medium passes to $E$ where it emerges in discrete drops from a calibrated jet and then, after running down the side of an inclined wide-bore tube through the fixed sealing head $D$, drips into the growth vessel. 
The growth vessel and fixed head

The growth vessel (Fig. 3, and $F$ in Fig. 2) is a cardioid-shaped flask of about $500 \mathrm{ml}$. capacity, having a broad neck with two concentric walls enclosing a 2:1 (v/v) glycerol + water mixture which seals it to the fixed head. It is mounted on a turntable, inclined at $30^{\circ}$ from the vertical, so that it can be rotated about its long axis at constant speed. The drive is provided by a rheostat-variable $24, \mathrm{~V}$., d.c. motor geared down to $400 \mathrm{rev} . / \mathrm{min}$. maximum speed. The fixed sealing-head is a glass or stainless-steel cylinder, open at the

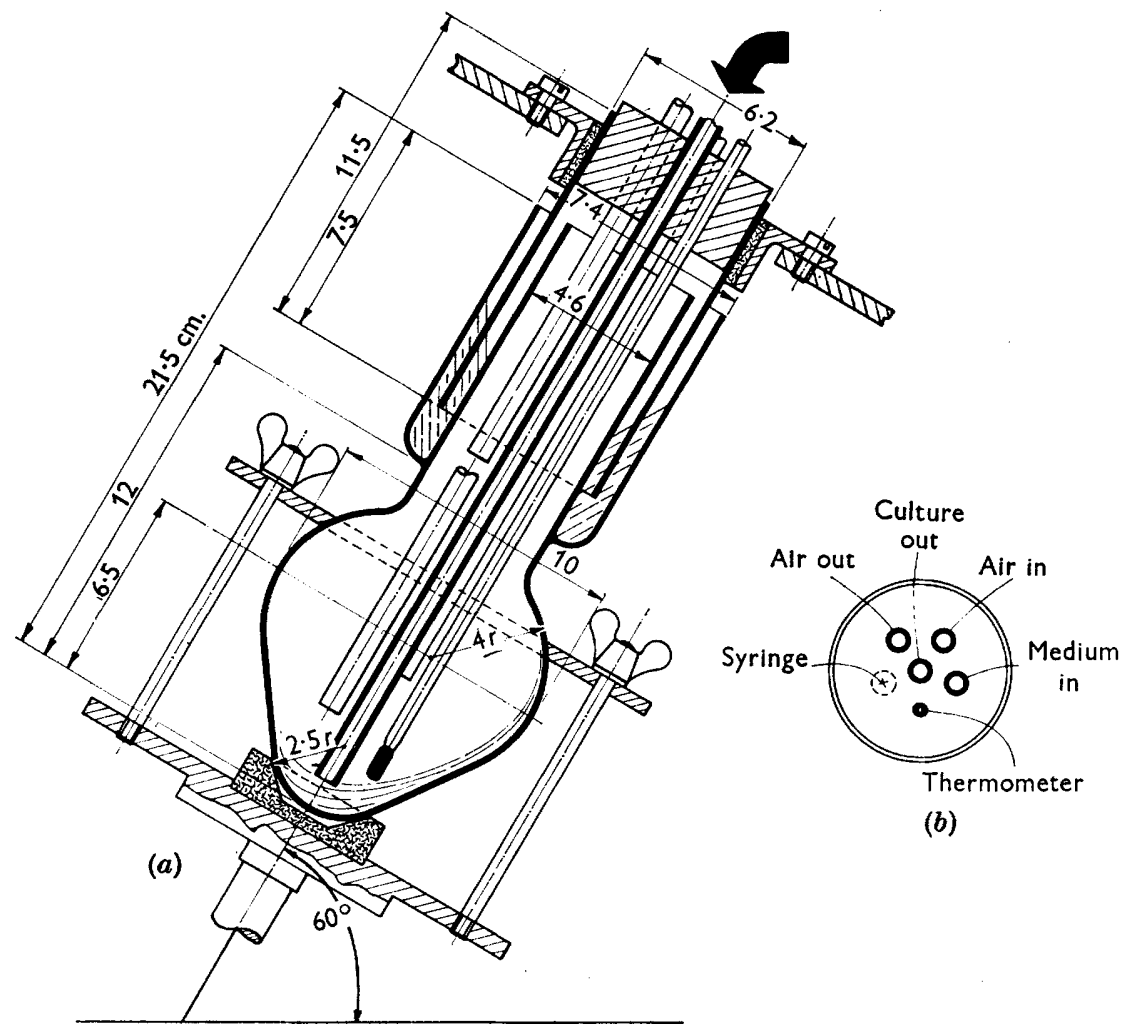

Fig. 3. (a) The growth vessel and parts of the associated equipment. The sealing-head shown is of the earliest type, being a glass cylinder closed at the top with a synthetic-rubber bung. (b) Plan of the top of the sealing-head, showing the disposition of the various apertures.

base and closed at the top except for a set of tubular sleeves, through which pass the various service tubes gripped by short lengths of rubber or silicone tubing. There is also an inoculation port stoppered with a soft-rubber cap to permit the passage of the needle of a hypodermic syringe. The fixed head is mounted on an adjustable gantry so that its long axis can be made to coincide with that of the spinning growth vessel.

During operation the growth vessel contains about $100 \mathrm{ml}$. of culture spun into a layer on the walls and base of the flask. Very good mixing results from the continual rapid transport of culture, once in each revolution, from top to 
bottom of the flask. Drops of acid or alkali added to a solution of $\mathrm{pH}$ indicator in the flask appear to produce instantaneous colour changes throughout the liquid mass. There is no foaming even with the richest media, and the large surface area exposed provides aeration good enough to support cultures of Escherichia coli or Bacillus cereus equivalent to $1 \mathrm{mg}$. dry wt./ml. at a generation time of $c .25 \mathrm{~min}$.

\section{Culture disposal}

The volume of culture is maintained constant in the growth vessel, so long as dilution rate and speed of rotation are constant, by a capillary tube of about $1.5 \mathrm{~mm}$. bore passing through the centre of the fixed head to within about $1.5 \mathrm{~cm}$. of the bottom of the vessel and connected indirectly to the negative air line. The rapid low-amplitude wobble of the culture surface causes the tip of the capillary to be immersed for a very short period many times a minute. As a result, a continual stream of air interspersed with occasional beads of culture is drawn rapidly up the tube and normally travels through tap $T_{3}$ to the waste receiver $I$, where the culture drips into the lysol and the air is withdrawn through filter $J$ into the vacuum source.

When a sample of the culture is required a tube is attached to the groundglass cone of the sampling head $H$; $\operatorname{tap} T_{4}$ is opened, to connect the tube to the negative air line, and tap $T_{3}$ is turned so that the effluent stream of air and culture is diverted from the waste receiver to the sampling head. The average time of transport of culture from growth vessel to sampling tube is less than $2 \mathrm{sec}$. The sampling tube can be surrounded by a thermos flask containing a suitable cooling or freezing mixture.

\section{Safety switch}

Most failures of the apparatus, other than breakages, lead only to a spoilt experiment. But if the suction pressure in the negative air line becomes inadequate while the apparatus is unattended the growth vessel may fill with culture, overflow through the liquid seal, and contaminate the hot room. To prevent that, manometer $K$ acts as a mercury switch in the circuit of a pulsed motor (Fig. 4) connected to tap $T_{2}$. When the suction falls below a safe limit the motor turns $T_{2}$ to the fully-off position, stopping the medium supply.

\section{COMMENTS ON THE DESIGN AND OPERATING CONDITIONS}

\section{Air supply}

Any sources of compressed air and vacuum are suitable if precautions are taken to prevent dangerously high or low pressures building up in the apparatus during faulty operation. The exact pressures and flow rates required in the air circuits depend mainly on the dimensions of the apparatus. In the prototype the positive air line supplied air at about $500 \mathrm{ml} . / \mathrm{min}$. and $+5 \mathrm{~cm}$. $\mathrm{Hg}$ pressure, and the negative line withdrew air at about $450 \mathrm{ml} . / \mathrm{min}$. and $-10 \mathrm{~cm} . \mathbf{H g}$ pressure. Both lines were served by a dual purpose bellows pump driven by a $\frac{1}{8}$ h.p. induction motor geared down to $60 \mathrm{rev} . / \mathrm{min}$. The pumping unit 
consisted of two flexible metal bellows connected to the common crankshaft by levers and connecting rods. The position of the fulcrum of each lever was separately adjustable, making the stroke lengths of the bellows independently variable. By this means it was easy to ensure that the volume of air blown into
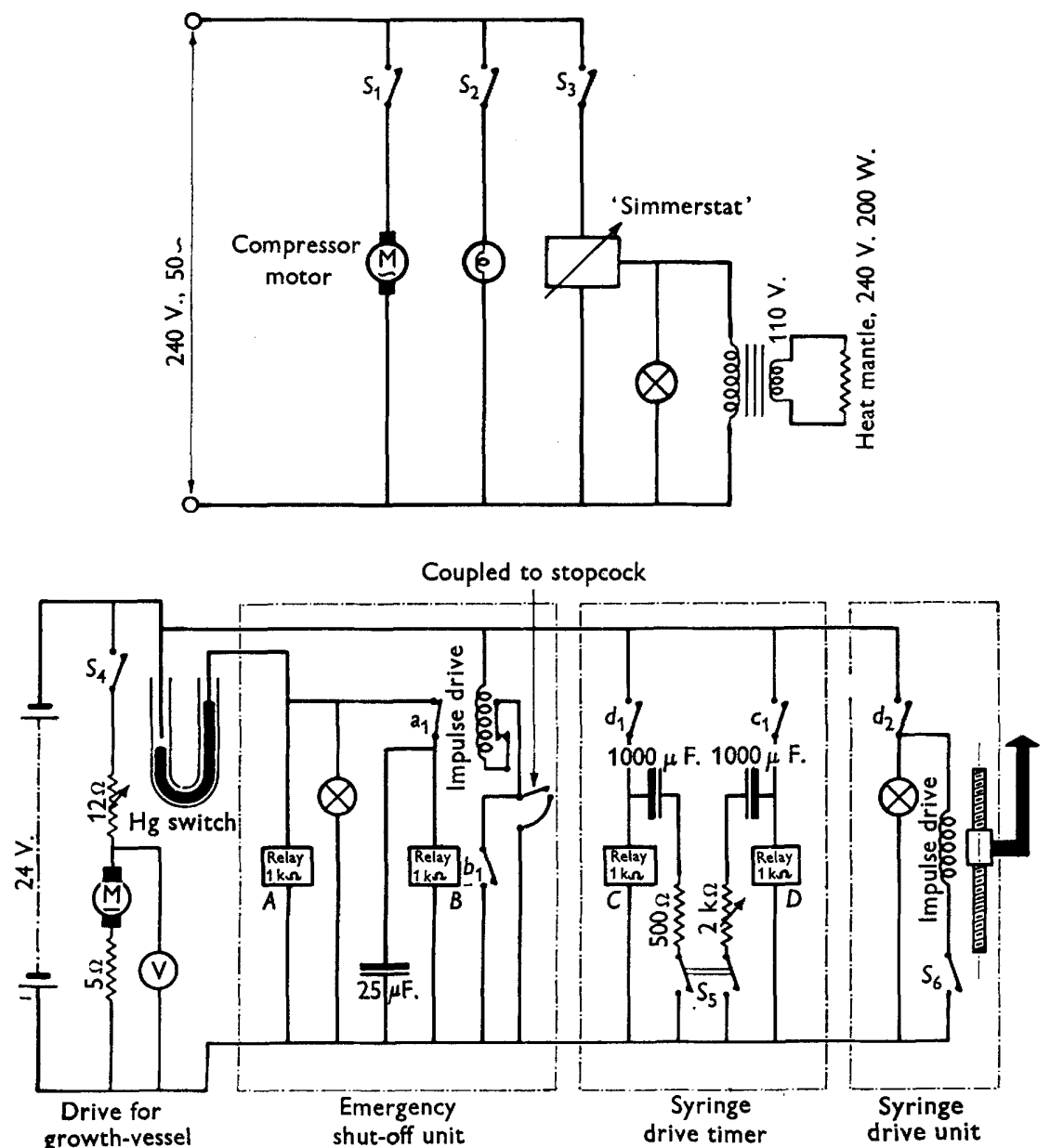

Fig. 4. The two electrical circuits associated with the apparatus, one operating at 230 V., A.C. and the other at 24 V., D.c.

the apparatus was always greater than the volume sucked out, so that air was never drawn into the growth vessel through filter $G$. The relative inefficiency of the ball-valves prevented the accidental build-up of very high or low pressures.

\section{Medium supply}

Silicone tubing (Dunlop Rubber Co. Ltd., Cambridge Street, Manchester, 1) was included in the medium flow lines as soon as it became available in order to increase the flexibility of the assembly. The silicone grease lubricating the 
ground-glass joints made the apparatus rather difficult to clean, and it appears that its replacement by 'Teflon' sleeves (Loughborough Glass Co. Ltd., Loughborough, Leicestershire) may be a great improvement.

\section{Air saturator}

The usual method of humidifying air by bubbling it through water at room temperature is inefficient unless the bubbles are very small or their time of contact with the water very long. With rapid flow rates it is simplest partially to saturate the air at a high temperature and then to cool it so that condensation occurs. When the condenser is long enough the emergent air is $100 \%$ saturated at ambient temperature. An air saturator is not absolutely essential, but it is advisable when very low growth rates are contemplated. For reasons discussed later the air-flow through the apparatus is considerably faster than is needed for adequate aeration. At $35^{\circ}$ an increase in saturation from 50 to $100 \%$ involves the removal of c. $1 \mathrm{ml}$. water $/ \mathrm{hr}$. for each 1 . air $/ \mathrm{min}$. When unsaturated air is used the effective concentration of the incoming medium will be greater than its made-up concentration and suitable corrections must be applied. In addition, unsaturated air will remove water from $\operatorname{trap} L$ and thereby alter the rate at which medium flows to the growth vessel. This effect is discussed in the next section.

\section{Constant-head vessel}

When a wide range of flow rates is required a constant-head variableresistance system has practical advantages over one with a variable-head and constant-resistance.

In the prototype medium flows under a head of $c .30 \mathrm{~cm}$. water, which is the sum of the depth of immersion of the tip of the bubbling tube in trap $L$, and the vertical distance between the tip of the bubbling tube in $B$ and the tip of the dropping-jet in $E$ (assuming that the density of the medium is unity). The contribution of air pressure to the total head may be increased at will by increasing the depth of immersion of the bubbling tube in $L$. Whatever the depth of water in $L$, in the absence of an air-saturator the apparatus must include some device for keeping it topped-up to constant level, or the medium flow rate will change progressively during operation.

It may sometimes be desirable to add a special metabolite at constant concentration over an extended period to a culture which has attained constant population density in its absence. This is done by means of the pre-mixing device without contaminating the main medium reservoir and without altering the culture dilution rate. The high-speed pulser is first used to inject into $B$ enough metabolite to raise the concentration to the desired level. The pulser is then switched to variable speed and set at whatever rate is necessary, relative to the culture dilution rate, in order to keep the concentration constant. At the same time some of the metabolite may be added directly to the growth vessel through the inoculation port. Tap $T_{1}$ provides a constriction in the medium line which prevents the special metabolite from diffusing into the main reservoir against the stream of medium. At the end of an addition 
period the pulser is switched off, $B$ is drained through $T_{1}$ and allowed to refill at once with fresh medium.

As an improvement, the inlet capillary from $C$ is led into $B$ towards the bottom of the vessel, instead of at the top, to avoid the possible disturbing effect of suction on the motion of the syringe plunger. In addition, the capillary is provided with a 3-way tap so that the syringe can be refilled without disconnecting it.

\section{Variable flow resistance}

The grooved $\operatorname{tap} T_{2}$ gave satisfactory control in the prototype at the faster dilution rates, provided it was mounted with the wide groove uppermost (Fig. 5) to prevent small air bubbles forming partial blockages. A great improvement is the substitution for $T_{2}$ of an array of six precision-bore capillary tubes ('Veridia' from Chance Bros. Ltd., Smethwick 40, Birmingham), mounted in parallel, giving flow-rates in the ratios $1: 2: 4: 8: 16: 32$. By means of taps the capillaries can be included in the medium circuit, individually or in all combinations, giving a range of 63 different dilution times between 20 min. and $21 \mathrm{hr}$. For this purpose long wide capillaries are preferred to short fine ones, being less likely to become clogged. The longer tubes are coiled to save space.

The efficacy of any type of flow resistance depends on the viscosity of the medium: the viscosity of water varies with temperature by nearly $2 \%$ per degree in the vicinity of $35^{\circ}$. It is therefore important that the temperature of the medium supply system be kept constant, and medium used for replenishing the reservoir should always be brought to hot room temperature before addition.

\section{The growth vessel}

When the vessel is spun too fast the culture leaves the bottom and clings to the sides in a smooth band. For best aeration and mixing conditions the speed used should be the highest at which a layer of culture remains always on the bottom of the vessel. In the prototype about $300 \mathrm{rev} . / \mathrm{min}$. was used, but larger vessels must be rotated at slower speeds.

The volume of cultures contained in the growth vessel diminishes slightly as the dilution rate is decreased. Since it is impracticable to measure the volume of culture contained in the vessel while it is spinning, dilution rates are determined accurately by injecting into the vessel ${ }^{32} \mathbf{P}$ (as phosphate) and measuring the radioactivity of samples collected at intervals. The straight line plot of log. radioactivity against time has a slope corresponding to the dilution rate. This procedure also eliminates errors which might arise through changes in the size of drop delivered by the jet in $E$, due to different media having different surface tensions.

One of the main disadvantages of using this type of growth vessel is that the design cannot be 'scaled-up' many times without decreasing the efficiency of aeration. But an alternative vessel of 2 l. capacity accommodating $400 \mathrm{ml}$. of culture has been included in a later model for use at low growth rates. 


\section{Culture disposal}

It is theoretically possible to keep the culture volume constant indirectly by some device such as a double bellows pump which removes culture at the same rate as it adds medium. But it is practically impossible to ensure that the two pumping rates are absolutely identical, and any difference between them will have a cumulative effect. The volume of culture in the growth vessel, and therefore its growth rate, will then progressively increase or decrease throughout a run.

\section{Electrical equipment}

The use of $24 \mathrm{~V}$., D.c. components in many of the circuits was dictated by the economic need to use cheap war-surplus equipment. But the current consumption at that voltage is inconveniently high, and the use of components with a much higher voltage rating is to be preferred.

\section{FACTORS WHICH GOVERN THE DIMENSIONS OF THE APPARATUS}

The identity of the organism, the temperature at which it is to be grown and the proportional composition of the medium (which may be simple or complex, and with or without a known limiting nutrient) having been decided, it is then necessary to determine three properties of the system: (1) $\gamma_{M}$, the maximum growth rate of the organism in the presence of an excess concentration of medium, expressed as doublings of dry wt./hr.; (2) $Y$, the dry wt. yield of organism per unit mass of nutrient consumed; (3) the curve relating growth rate and medium concentration, which will usually be of the general form shown in Fig. 6. A detailed and lucid discussion of the best way to determine these properties in batch culture appears in the publications of Monod (1942, 1949) which should be read by anyone contemplating studies on bacterial growth. There is now evidence, good in the case of Aerobacter cloacae (Herbert et al. 1956) and less convincing in the case of Escherichia coli (Perret, unpublished) that under some conditions $Y$ may decrease at growth rates near to $\gamma_{B K}$. But it is assumed here that, at a fixed temperature, $\boldsymbol{Y}$ is a constant independent of growth rate. It is also assumed that the relationship between growth rate and nutrient concentration depicted in Fig. 6 is independent of the previous history of the organisms, although theoretical and experimental evidence show that the assumption cannot be true. In fact, the curve in Fig. 6 strictly holds only for bacteria which are being subjected to changes of nutrient concentration at an infinitely slow rate, so that some of the published mathematical theory of continuous culture is misleading; this may have an important bearing on practical attempts to maintain bacteria at very low growth rates. It is hoped to discuss this discrepancy at length elsewhere, but for the present purpose of providing rough practical guidance in design the assumptions made are good enough. 


\section{Medium concentration}

For metabolic studies it is advisable that the medium concentration, and therefore the amount of organisms/unit volume, should be as low as possible. The reasons for this appear later.

Let $\gamma_{1}$ be the fastest growth rate which it is desired to attain in the apparatus $\left(\gamma_{1}<\gamma_{M}\right)$ and let $W \mathrm{mg} . / \mathrm{ml}$. be the lowest concentration of bacteria grown at
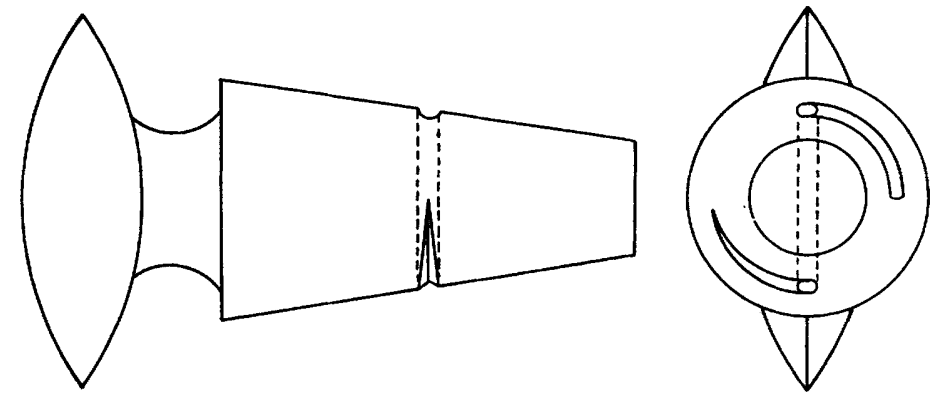

Fig. 5

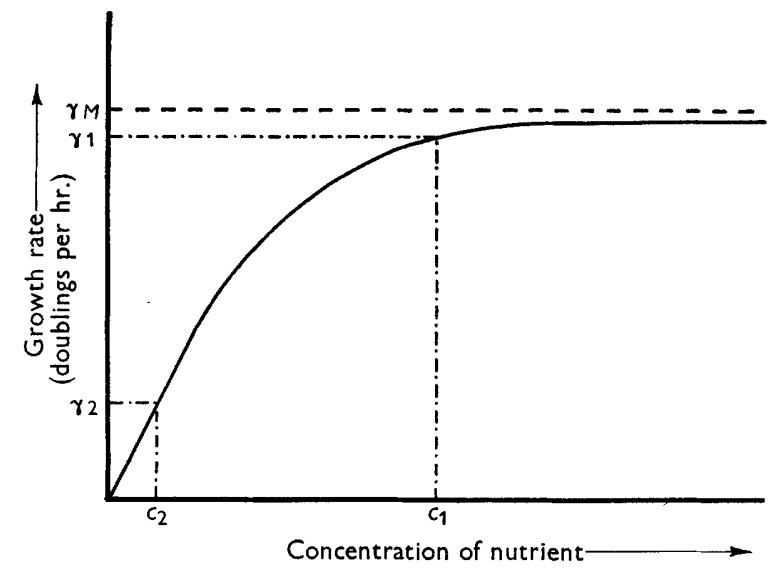

Fig. 6

Fig. 5. Details of the grooved taps used in the prototype. For satisfactory control of flowing liquids the taps must be mounted with the wide groove uppermost, so that air bubbles entering from below are not trapped.

Fig. 6. A portion of the type of curve relating growth rate and nutrient concentration. For most limiting nutrients the curve has the same general form, but the actual nutrient concentration scale may vary over a many thousand-fold range. $\gamma_{M}$ is the maximum growth rate attained in the presence of an excess concentration of nutrient.

$\gamma_{1}$, which will be acceptable for the experiments planned. The concentration of unconsumed nutrient in the effluent culture at $\gamma_{1}$ will be the same as $c_{1}$, the ambient concentration in the growth vessel (Fig. 6). $C$, the lowest acceptable concentration of the incoming medium is therefore fixed by the equation

$$
C=\frac{W}{Y}+c_{1} \text {. }
$$




\section{Culture volume}

In general, the larger the culture volume the better. For economy in medium consumption, however, it may be necessary to decide how small the capacity of the growth vessel can safely be made. No precise answer is possible: it is ultimately a matter of opinion. But by making a few simple calculations it can at least become a matter of enlightened opinion. The minimum volume of culture is set by practical rather than theoretical factors. In order to avoid growth of bacteria up the medium inflow tube into the main reservoir it is necessary either to break the liquid continuity between sterile medium and culture, or always to have an inflow velocity so great that the bacteria cannot possibly migrate against the stream. A continuous jet of medium crossing an air gap provides the ideal solution, but it entails very high flow rates and correspondingly large culture volumes. The usual solution is to add the medium in discrete drops at regular intervals. Such discontinuous addition is in principle unsound, since it must lead to fluctuations of nutrient concentration in the culture. The higher the frequency of the concentration swing, and the lower its amplitude, the nearer the system approaches to its ideal state. Our knowledge is at present inadequate to prove that a given type of fluctuation is of negligible significance; one can merely say that it appears unlikely to be important in a particular context. Each worker must therefore decide for himself what fluctuations he is prepared to regard as unimportant for his purpose.

It can be seen from Fig. 6 that concentration changes have their greatest effect at the lowest growth rates. If $\gamma_{2}$ is the lowest growth rate to be attained in the apparatus and $c_{2}$ the corresponding ambient nutrient concentration, let it be decided that the increase in nutrient concentration following the addition of one drop of medium must not be greater than $\alpha c_{2}$, and that the frequency of the fluctuation must not be less than $\beta / \mathrm{hr}$. Then if $v$ is the volume of a single drop of medium

$$
V_{1}=\frac{v C}{\alpha c_{2}}
$$

and

$$
V_{2}=\frac{v \beta}{\log _{e} 2 \cdot \gamma_{2}}
$$

where $V_{1}$ and $V_{2}$ are respectively the lowest culture volumes to fulfil the fluctuation amplitude and frequency provisos.

A third factor which affects the culture volume is the size of sample necessary for the proposed investigations. If the volume of a sample is a very small proportion of the volume of culture in the growth vessel it may be removed directly without seriously upsetting the system. But if the sample volume is of relatively significant size it must be obtained by collecting the effluent culture continuously being withdrawn through the constant volume control tube. The time required for collecting a sample of given volume will then be proportional to the dilution rate. When the constancy of conditions in the growth vessel is not in question the composition of a sample will be the same, whether it be 
obtained quickly or slowly. But when the constancy of conditions is in question, samples must be collected over a period short enough to reveal possible trends or cyclical variations. If the volume of a sample must be $x \mathrm{ml}$. and the maximum time to be allowed for collecting it is $t \mathrm{hr}$. when the culture is at its minimum growth rate $\gamma_{2}$, then the volume of culture in the growth vessel must not be less than $V_{3}$

$$
V_{3}=\frac{x}{\log _{e} 2 \cdot \gamma_{2} t}
$$

To satisfy all the conditions the volume of culture in the growth vessel must be at least equal to whichever is the largest of $V_{1}, V_{2}$ and $V_{3}$. As an example consider the case of Escherichia coli which is to be grown on a glucose-limited defined medium at a minimum density $W$ of $1 \mathrm{mg} . / \mathrm{ml}$., a maximum growth rate $\gamma_{1}$ of 1.25 (in population doublings per hr.) and a minimum growth rate $\gamma_{2}$ of $0 \cdot 2$. Let the highest acceptable concentration fluctuation be arbitrarily fixed as $0.5 c_{2}$ at a lowest frequency of $300 / \mathrm{hr}$. The size of a drop of medium is $0.05 \mathrm{ml}$., and it is required to collect $2 \mathrm{ml}$. samples in not more than $0.15 \mathrm{hr}$. From the published results of Monod (1942), $Y=c .0 \cdot 23, c_{1}=c .0 .04 \mathrm{mg} . / \mathrm{ml}$. and $c_{2}=c .0 .002 \mathrm{mg} . / \mathrm{ml}$. Then from equations (1)-(4): $C=4.4 \mathrm{mg} . / \mathrm{ml}$, $V_{1}=220 \mathrm{ml}$., $V_{2}=108 \mathrm{ml}$. and $V_{3}=96 \mathrm{ml}$, in which case the overriding value for the culture volumes is $V_{1}$ at $220 \mathrm{ml}$.

\section{Medium, culture and air flow rates}

The volume of culture, $V$, having been decided, then the maximum flow rate $F_{1}$ at which medium must be delivered to the growth vessel is easily calculated

$$
F_{1}=\gamma_{1} V \log _{e} 2 \mathrm{ml} \text {. } / \mathrm{hr} .,
$$

and the relationships between constant head and flow-resistance follow from Poiseuille's Law.

The constant-volume control system must obviously be able to remove culture at not less than $F_{1} \mathrm{ml} . / \mathrm{hr}$. But at high growth rates, if that minimum value is chosen, the sampling tube will be almost continuously full of culture moving at a comparatively slow rate, under anaerobic conditions, towards the sampling head. The effluent culture may then undergo such changes that samples no longer closely represent the composition of the main culture. The extent of the changes will depend on the density of the culture and on the time spent in transit. Since the culture density has already been set as low as possible in order to keep $V$ small, it only remains possible to shorten transit time. This can be done by making the sampling tube of the narrowest capillary not likely to become blocked, and using a suction pressure at which the potential flow-rate of culture is much greater than $F_{1}$. Droplets of effluent culture will then be swept rapidly to the sampling head by a high velocity air stream. At $35^{\circ}$ water is about 40 times as viscous as air; so for a potential culture extraction rate of $n F_{1}$, sterile air must be supplied to the apparatus at a rate of about $40 n F_{1}$ if negative pressure is not to develop in the growth vessel at low growth rates. 
The author is considerably indebted to the staff of the Engineering Division of the National Institute for Medical Research, and to Mr N. Schunmann, for their expert and patient assistance during the construction of the original apparatus.

\section{REFERENCES}

De HAan, P. G. \& Winkler, K. C. (1955). An apparatus for the continuous culture of bacteria at constant generation times. Leeurvenhoek ned. Tijdschr. 21, 33.

Herbert, D., Elsworth, R. \& Telling, R. C. (1956). The continuous culture of bacteria: a theoretical and experimental study. J. gen. Microbiol. 14, 601.

Monod, J. (1942). Recherches sur la croissance des cultures bactériennes. Paris: Hermann et Cie.

Monod, J. (1949). The growth of bacterial cultures. Annu. Rev. Microbiol. 3, 371.

Monod, J. (1950). La technique de culture continue: théorie et applications. Ann. Inst. Pasteur, 79, 390.

Novick, A. (1955). Growth of bacteria. Annu. Rev. Microbiol. 9, 97.

Novick, A. \& Szilard, L. (1950a). Description of the Chemostat. Science, 112, 715.

Novick, A. \& Szilard, L. (1950b). Experiments with the Chemostat on spontaneous mutations of bacteria. Proc. nat. Acad. Sci., Wash. 36, 708.

SPICER, C. C. (1955). The theory of bacterial constant growth apparatus. Biometrics, $11,225$. 